\title{
ON QUASI-PERMUTATION REPRESENTATIONS OF FINITE GROUPS
}

\author{
by J. M. BURNS, B. GOLDSMITH, B. HARTLEY and R. SANDLING
}

(Received 5 January, 1993)

1. Introduction. In [6], Wong defined a quasi-permutation group of degree $n$ to be a finite group $G$ of automorphisms of an $n$-dimensional complex vector space such that every element of $G$ has non-negative integral trace. The terminology derives from the fact that if $G$ is a finite group of permutations of a set $\Omega$ of size $n$, and we think of $G$ as acting on the complex vector space with basis $\Omega$, then the trace of an element $g \in G$ is equal to the number of points of $\Omega$ fixed by $g$. In [6] and [7], Wong studied the extent to which some facts about permutation groups generalize to the quasi-permutation group situation. Here we investigate further the analogy between permutation groups and quasipermutation groups by studying the relation between the minimal degree of a faithful permutation representation of a given finite group $G$ and the minimal degree of a faithful quasi-permutation representation. We shall often prefer to work over the rational field rather than the complex field.

By a quasi-permutation matrix we mean a square matrix over the complex field $\mathbb{C}$ with non-negative integral trace. Thus, every permutation matrix over $\mathbb{C}$ is a quasipermutation matrix. For a given finite group $G$, let $p(G)$ denote the minimal degree of a faithful permutation representation of $G$ (or of a faithful representation of $G$ by permutation matrices), let $q(G)$ denote the minimal degree of a faithful representation of $G$ by quasi-permutation matrices over the rational field $\mathbb{Q}$, and let $c(G)$ be the minimal degree of a faithful representation of $G$ by complex quasi-permutation matrices. Thus,

$$
c(G) \leq q(G) \leq p(G) .
$$

It is easy to see that if $G$ is cyclic of order 6 , then $c(G)=q(G)=4$ and $p(G)=5$, while on the other hand, if $G$ is the quaternion group of order 8 , then $c(G)=4$ and $q(G)=$ $p(G)=8$. Thus, both inequalities can be strict. It is not too hard to see that for the group $\operatorname{SL}(2,5)$, both inequalities are strict (see Section 4). Our principal aim in this paper is to investigate these quantities and inequalities further, and our main theorem characterizes those finite abelian groups for which equality holds.

TheOREM A. Let $G$ be finite abelian. Then $c(G)=q(G)$, and $q(G)=p(G)$ if and only if $G$ has no direct factor of order 6.

If $G$ is a finite abelian group and $G=G_{1} \times \ldots \times G_{r}$, where each factor is a non-trivial cyclic group of prime power order, then the trace of $G$ is defined by Hoffman [3] to be $T(G)=\sum_{i=1}^{r}\left|G_{i}\right|$. We modify Hoffman's definition slightly by putting $T(1)=1$. It is then not hard to see that $T(G)=p(G)[3]$. It does not seem completely straightforward to find groups $G$ of odd order for which $q(G)<p(G)$. We show how to calculate these quantities for groups of the form $G=A B$, where $A$ is an elementary abelian minimal normal $p$-subgroup of $G$ and $B$ is a cyclic subgroup of prime order $q$. In order to show that this

Glasgow Math. J. 36 (1994) 301-308. 
gives rise to a group $G$ of odd order for which $q(G)$ differs from $p(G)$, we have had to use CAYLEY [1], though in a somewhat indirect manner.

Part of this work is the result of a visit of the middle two authors to the Dipartimento di Matematica Pura ed Applicata, Università di Padova. They express their gratitude to Professors Menegazzo, Salce and Zacher in particular, as well as numerous other colleagues in Padova, for splendid hospitality.

2. Transitivity. If $G$ is any group and $K$ any field of characteristic zero, we call a $K G$-module $V$ a permutation module if it affords a representation by permutation matrices, and a quasi-permutation module if it affords a representation by quasipermutation matrices, that is, matrices of non-negative integral trace. We write $\chi_{v}$ for the character of a $K G$-module $V$. A simple property of permutation modules is that they contain the trivial module; this extends to the quasi-permutation situation.

Lemma 2.1. If $G$ is a finite group and $V$ is a quasi-permutation module for $G$ over $K$, then $V$ contains a trivial $K G$-module of dimension at least one.

Proof. We may take $V$ to be faithful for $G$ and identify $G$ with a group of linear transformations of $V$. Let $\alpha=\sum_{g \in G} g$. Since the identity has trace $n$ and the trace $\operatorname{Tr}(g)$ of each element $g \in G$ is non-negative, we have $\operatorname{Tr}(\alpha) \geq n$. In particular, $\alpha \neq 0$. Choosing $v \in V$ such that $\alpha v \neq 0$, we have that $\alpha v$ generates a trivial module.

If $G$ is a finite permutation group with $t$ transitive constituents and we form the corresponding permutation module $V$, then a well known theorem of Burnside asserts that $V$ contains the trivial module with multiplicity exactly $t$. Otherwise stated, $\sum_{g \in G} \operatorname{Tr}(g)=t|G|$. We call a quasi-permutation module indecomposable if it cannot be written as the direct sum of two non-zero quasi-permutation submodules. We may consider these to be somewhat analogous to transitive permutation representations, though it is not clear how good the analogy is. As an immediate consequence of Lemma 2.1 , we have the following.

LemMa 2.2. Let $V$ be a quasi-permutation module for $G$ and suppose that $V=$ $V_{1} \oplus \ldots \oplus V_{t}$, where the $V_{i}$ are quasi-permutation submodules of $V$. Then $V$ contains the trivial module with multiplicity at least $t$.

The following simple example shows that equality need not occur. Let $G$ be cyclic of order $p q$, where $p$ and $q$ are distinct primes. Let $V$ be a faithful irreducible module for $G$ over the rationals. Then (see Lemma 3.4) $\operatorname{dim} V=(p-1)(q-1)$, and if $\chi_{v}$ is the character of $V$ and $x \in G$, then $\chi_{V}(x)=(p-1)(q-1),-(q-1),-(p-1), 1$ according as the order of $x$ is $1, p, q, p q$ respectively. Suppose that $p>q$ and let $T$ be a trivial $G$-module of dimension $p-1$. Then $V \oplus T$ is an indecomposable quasi-permutation module containing the trivial module with multiplicity $p-1$.

COROLlaRy 2.3. Let $V$ be a permutation module corresponding to a transitive permutation representation. Then $V$ is indecomposable as quasi-permutation module.

Proof. This is because $V$ contains the trivial module with multiplicity one. 
3. The minimal degree of a faithful quasi-permutation representation of an abelian group. We begin with the only positive result we have in the non-abelian case.

Proposition 3.1. Let $G$ be a finite p-group, let $K$ be a field of characteristic zero, and let $V$ be a faithful quasi-permutation module for $G$ of minimal dimension. Then $p \mid \operatorname{dim} V$.

Proof. By Lemma 2.1 and Maschke's Theorem, we have $V=W \oplus T$, where $T$ is a one-dimensional trivial module and $W$ is a $K G$-module which is clearly faithful. Hence, by the minimality, $W$ is not a quasi-permutation module, and so $\chi_{W}(x)<0$ for some $x \in G$. Since $\chi_{V}(x)=1+\chi_{W}(x)$ is a non-negative integer, we must have $\chi_{V}(x)=0$. But if $\operatorname{dim} V=n$, then $\chi_{v}(x)$ is a sum of $n p^{\alpha}$-th roots of 1 , where $|G|=p^{\alpha}$. By [6, Lemma 1(iv)], we have $\chi_{v}(x) \equiv n(\bmod p)$, which gives the claimed result.

Now we prepare for the proof of our main theorem on finite abelian groups. First we note the following well known fact about the Schur index.

Lemma 3.2. Suppose that $G$ has the property that the Schur index over the rationals of every irreducible character of $G$ is 1 , and let $\chi$ be any rational valued character of $G$. Then $\chi$ is the character of a representation of $G$ over $\mathbb{Q}$. In particular, $c(G)=q(G)$.

Proof. Let $\zeta$ be a primitive $|G|$-th root of 1 , let $K=\mathbb{Q}(\zeta)$, and $\Gamma$ be the Galois group of $K$ over $\mathbb{Q}$. If $\theta$ is any irreducible complex character of $G$, then $\theta$ takes its values in $K$, and our hypothesis implies that the sum of the distinct $\Gamma$-conjugates of $\theta$ is the character of a representation of $G$ over $\mathbb{Q}\left[4\right.$, Corollary 10.2]. If $\left\{\theta_{i}: 1 \leq i \leq m\right\}$ is the set of irreducible characters of $G$ and $\chi=\sum_{i=1}^{m} r_{i} \theta_{i}$ with non-negative integral coefficients $r_{i}$, then because of the $\Gamma$-invariance of $\chi$, we have $r_{i}=r_{j}$ whenever $\theta_{i}$ and $\theta_{j}$ are $\Gamma$-conjugate. In other words, $\chi$ is a non-negative integral combination of $\Gamma$-orbit sums of irreducible characters, and so it is the character of a rational representation.

Corollary 3.3. If $G$ is finite abelian, then $c(G)=q(G)$.

Proof. This is because $G$ satisfies the hypothesis of Lemma 3.2 [4, Lemma 10.8].

Now we recall some well known facts about irreducible representations of finite abelian groups over $\mathbb{Q}$. Let $A$ be finite abelian, let $V$ be an irreducible $\mathbb{Q} A$-module, and let $K=C_{A}(V)$ be the kernel of the representation of $A$ on $V$. Then $A / K$ is cyclic, and so for many purposes we only need to consider irreducible representations of cyclic groups. Suppose then that $A=\langle a\rangle$ is cyclic of order $m$. Then for each $d \mid m$, there is an irreducible $\mathbb{Q} A$-module $V(d)$ of dimension $\phi(d)$, where $\phi$ is the Euler totient function. We can take $V(d)$ to be $\mathbb{Q}\left(\zeta_{d}\right)$, where $\zeta_{d}$ is a primitive $d$-th root of unity, and $a$ acts on $V(d)$ as multiplication by $\zeta_{d}$. Since $\sum_{d \mid m} \phi(d)=m$, the modules $V(d)$ are, up to isomorphism, all the irreducible $\mathbb{Q} A$-modules. Thus, there is exactly one for each divisor $d$ of $m$. Let $\chi_{d}$ denote the character of $V(d)$, for brevity. Then $\chi_{d}(a)$ is the sum of the primitive $d$-th roots of unity, and so is equal to $\mu(d)$, where $\mu$ is the Möbius function.

Lemma 3.4. Let $A$ be cyclic of order $m$, and let $b$ be an element of $A$ of order $d \mid m$. Then $\chi_{m}(b)=(\phi(m) / \phi(d)) \mu(d)$.

Proof. As a module for $\langle b\rangle, V(m)$ is a direct sum of faithful irreducible modules. By 
the above remarks, these have dimension $\phi(d)$, and $b$ has trace $\mu(d)$ on each of them. Further, $\operatorname{dim} V(m)=\phi(m)$. From these statements the result follows.

Now we need some inequalities involving the Euler totient function. If $n$ is a natural number and $n=p_{1}^{r_{1}} \ldots p_{t}^{r_{1}}$, where the $p_{i}$ are distinct primes, we write $n^{*}=\sum_{i=1}^{t} p_{i}^{r_{i}}$. Thus, if $G$ is a finite cyclic group, then $|G|^{*}=T(G)$.

LeMmA 3.5.(i) Let $m$ be a positive integer. Then $2 \phi(m) \geq m^{*}$, unless $m=6$, when $2 \phi(m)=4$.

(ii) Let $m=2^{\alpha} n$, where $a \geq 0$ and $n$ is odd. Then $(p / p-1) \phi(m) \geq n^{*}$ for each prime divisor $p$ of $n$.

Proof. (i) If $m=\prod_{i=1}^{k} p_{i}^{\alpha_{i}}$, where $\alpha_{i}>0$, then

$$
2 \phi(m)=2 \prod p_{i}^{\alpha_{i}-1}\left(p_{i}-1\right) \geq 2 \sum p_{i}^{\alpha-1}\left(p_{i}-1\right)
$$

unless some $p_{i}^{\alpha_{i}-1}\left(p_{i}-1\right)=1$. Since $2\left(p_{i}-1\right) \geq p_{i}$, this gives the result unless some $p_{i}^{\alpha_{i}}$ is 2. In this case, taking $p_{1}^{\alpha_{1}}=2$, we have $2 \phi(m) \geq 2 \sum_{i=2}^{k} p_{i}^{\alpha_{i}-1}\left(p_{i}-1\right)$. Now $2\left(p_{i}-1\right) \geq p_{i}+2$ if $p_{i} \geq 5$, and this gives the result unless $m$ has the form $2.3^{\alpha}$, when it is easily checked.

(ii) In this case, if $a \geq 2$, then $\phi(m)=2^{a-1} \phi(n)=2^{a-2}(2 \phi(n)) \geq n^{*}$, by (i). Suppose then that $a \leq 1$. Then $\phi(m)=\phi(n)$. We check explicitly that the result holds in the cases $n=p^{r}, 3 p^{r}$, where $p$ is a prime. Otherwise we can write $n=x y$, where $(x, y)=1$ and both $\phi(x)$ and $\phi(y)$ are at least 4 . Using the inequality $u v \geq 2(u+v)$, which holds if $u, v \geq 4$, we obtain

$$
\frac{p}{p-1} \phi(n) \geq \phi(n)=\phi(x) \phi(y) \geq 2(\phi(x)+\phi(y)) \geq x^{*}+y^{*}=n^{*}
$$

by (i).

The next result is the core of our proof of Theorem $A$.

LEMMA 3.6. Let $G$ be a finite abelian group having no direct factor of order 6 , and let $V$ be a $\mathbb{Q} G$-module. Suppose that $V$ is faithful for $G$, but no proper submodule of $V$ is faithful for $G$. Then $G$ contains an element $g$ such that $\chi_{v}(g)<0$ and $\operatorname{dim} V-\chi_{v}(g) \geq$ $\operatorname{Tr}(G)$.

Proof. Let $V=V_{1} \oplus \ldots \oplus V_{s}$, where each $V_{i}$ is an irreducible $\mathbb{Q} G$-module, and let $K_{i}=C_{G}\left(V_{i}\right), K_{i}^{*}=\bigcap_{j \neq i} K_{j}$. Then, by hypothesis, $\bigcap_{i=1}^{s} K_{i}=1$, but $K_{i}^{*} \neq 1$ if $1 \leq i \leq s$. Let $G_{p}$ be the Sylow $p$-subgroup of $G$, and $K_{i, p}=K_{i} \cap G_{p}$. Among the subgroups $K_{i, 2}$, choose a subset of minimal size with respect to having trivial intersection, and renumber the $V_{i}$ so that these are the first $t$. Then $\bigcap_{i=1}^{t} K_{i, 2}=1$, and $\bigcap_{j \in X} K_{j, 2} \neq 1$ if $X \subset\{1, \ldots, s\}$ and $|X|<t$. We interpret the case $t=0$ as corresponding to $G_{2}=1$.

Let $x_{j}$ be an involution in $\bigcap\left\{K_{i, 2}: i=1, \ldots, t, i \neq j\right\}$ and $x=x_{1} \ldots x_{r}$. Then $x$ is an involution and acts as an involution on each of $V_{1}, \ldots, V_{i}$; therefore it acts as -1 on each 
of these modules. Now renumber the $V_{i}$ so that $V_{1}, \ldots, V_{u}$ are precisely those on which $x$ acts as -1 . Then $x$ acts trivially on $V_{u+1}, \ldots, V_{s}$. For $j=u+1, \ldots, s$, choose $x_{j}$ of prime order $p_{j}$ in $K_{j}^{*}$, and let $g=x x_{u+1} \ldots x_{s}=x_{1} \ldots x_{t} x_{u+1} \ldots x_{s}$. Thus, $g$ acts as -1 on each of $V_{1}, \ldots, V_{u}$ and as an element of order $p_{j}$ on $V_{j}$ if $u+1 \leq j \leq s$. By Lemma 3.4, $\chi_{v_{i}}(g)=-\operatorname{dim} V_{i}$ if $1 \leq i \leq u$, and $\chi_{v_{i}}(g)=-1 /\left(p_{i}-1\right) \operatorname{dim} V_{i}$ if $u+1 \leq i \leq s$. Hence we have $\chi_{v}(g)<0$, and

$$
\operatorname{dim} V-\chi_{v}(g)=2 \sum_{i=1}^{u} \operatorname{dim} V_{i}+\sum_{i=u+1}^{s}\left(1+\frac{1}{p_{i}-1}\right) \operatorname{dim} V_{i} .
$$

For $j=1, \ldots, u$, let $M_{j}=K_{j}$ if $\left|G / K_{j}\right| \neq 6$, and $M_{j}=G_{3} K_{j}$ if $\left|G / K_{j}\right|=6$. Also, for $j=u+1, \ldots, s$, let $M_{j}=G_{2} K_{j}$. If $\left|G / K_{j}\right|=6$ and $1 \leq j \leq u$, then $K_{j}$ contains $\Omega_{1}\left(G_{3}\right)=$ $\left\{z \in G_{3}: z^{3}=1\right\}$. Otherwise, as the involution $x$ lies outside $K_{j}$, there is a cyclic subgroup of $G$ of order 6 intersecting $K_{j}$ trivially and so complementing it, contrary to hypothesis. It follows that $\cap K_{j, 3}=1$, where we intersect over all $j$ between 1 and $s$ but omitting those for which $1 \leq j \leq u$ and $\left|G / K_{j}\right|=6$. Hence, $\bigcap_{j=1}^{s}\left(M_{j} \cap G_{3}\right)=1$. Since $\bigcap_{j=1}^{\prime} K_{j, 2}=1$, we also have $\bigcap_{j=1}^{s}\left(M_{j} \cap G_{2}\right)=1$. Hence

$$
\bigcap_{j=1}^{s} M_{j}=1 .
$$

Let $|G| K_{i} \mid=n_{i}$. We have $\operatorname{dim} V_{i}=\phi\left(n_{i}\right)$. By Lemma 3.5(i), $2 \operatorname{dim} V_{i} \geq n_{i}^{*}$ if $n_{i} \neq 6$, and if $n_{i}=6$, then $\phi\left(n_{i}\right)=2$. Thus, if $m_{i}=\left|G / M_{i}\right|$, then $2 \operatorname{dim} V_{i} \geq m_{i}^{*}$ for $1 \leq i \leq u$. For $u+1 \leq i \leq s$, we have, from Lemma 3.5(ii), that $\left(1+1 /\left(p_{i}-1\right)\right) \operatorname{dim} V_{i} \geq m_{i}^{*}$. Thus, from $(1)$,

$$
\operatorname{dim} V-\chi_{\nu}(g) \geq \sum_{i=1}^{s} m_{i}^{*}
$$

From (2), $G$ is a subgroup of a direct product of cyclic groups of orders $m_{1}, \ldots, m_{s}$. The trace of this direct product is the right hand side of (3). But the trace of a subgroup of a finite abelian group does not exceed the trace of the whole group, as can easily be seen directly, or from [3]. Therefore, the lemma follows from (3).

Proof of Theorem $A$. Since the equality $c(G)=q(G)$ follows from Lemma 3.2, we may restrict attention to $q(G)$ and $p(G)$. Suppose that $G$ has no direct factor of order 6 , and let $T$ be a $\mathbb{Q} G$-module affording a faithful quasi-permutation representation of minimal degree of $G$. Write $T=V \oplus W$, where $V$ is faithful for $G$, but no proper submodule of $V$ is faithful for $G$, and let $g$ be as in Lemma 3.6. Now by the remark following the statement of Theorem $\mathrm{A}, p(G)=T(G)$, and so Lemma 3.6 gives

$$
\operatorname{dim} V-\chi_{v}(g) \geq p(G)
$$

and $\chi_{V}(g)<0$. Since $\chi_{T}(g) \geq 0$, we have

$$
0 \leq \chi_{T}(g)=\chi_{v}(g)+\chi_{W}(g) \leq \operatorname{dim} V-p(G)+\operatorname{dim} W,
$$

whence $p(G) \leq \operatorname{dim} V+\operatorname{dim} W=\operatorname{dim} T=q(G)$. The reverse inequality is clear, and so Theorem $\mathrm{A}$ is established. 
4. Some non-abelian examples. The main aim of this section is to construct finite groups $G$ of odd order for which $q(G)<p(G)$. First we show how to calculate $c(G), q(G)$ and $p(G)$ for $G=S L(2,5)$. Since $G$ has a unique minimal normal subgroup, $p(G)$ is the smallest index of a subgroup with trivial core (that is, containing no non-trivial normal subgroup), and this is $120 / 5=24$. Note that $G$ contains exactly one involution $z$, which is therefore central and lies in every subgroup of even order.

We claim that $c(G)=8$ and $q(G)=16$. Thus, for this group,

$$
c(G)<q(G)<p(G) .
$$

To see these values, note that the smallest degree $d$ of a faithful rational character of $G$ is the smallest value of $\chi(1)+\chi^{\alpha}(1)+\ldots$, where $\chi$ is a faithful complex character of $G$ and $\left\{\chi, \chi^{\alpha}, \ldots\right\}$ is its orbit under the Galois group of $\mathbb{Q}(\chi)$ over $\mathbb{Q}$. From the character table of $G$, (see [2]), we see that $d=4$. The involution $z$ acts as -1 on this module, and we convert it to a quasi-permutation module by adding a trivial module of dimension 4 . This gives $c(G)=8$. The smallest degree $e$ of a faithful rational representation of $G$ is the smallest value of $m\left(\chi(1)+\chi^{\alpha}(1)+\ldots\right)$, where the notation is as above and $m$ is the Schur index of $\chi$ over $\mathbb{Q}$. From [5], where these Schur indices were calculated, we see that $e=8$. For the same reason as before we obtain a quasi-permutation module by adding a trivial module of dimension 8 , and find that $q(G)=16$.

Now let $G=A B$, where $B=\langle b\rangle$ is of prime order $q$, and $A$ is an elementary abelian normal $p$-subgroup of $G$ transformed faithfully and irreducibly by $B$. Thus, $p \neq q$. We show how to calculate $p(G)$ and $q(G)$ for groups of this type. We can think of $A$ as an $\mathbb{F}_{p} B$-module, with $B$ acting by conjugation. Let $n$ be the order of $p \bmod q$ and let $F$ be the field with $p^{n}$ elements. We can identify $A$ with the additive group of $F$, on which $b$ acts as multiplication by a primitive $q$-th root of 1 , say $\zeta$. First consider $p(G)$. If $\Omega$ is a $G$-set of minimal order on which $G$ acts faithfully, then $A$ acts non-trivially on some orbit of $\Omega$, and since $A$ is the unique minimal normal subgroup of $G$, that orbit is faithful. Thus, $\Omega$ is transitive. If $S$ is the stabilizer of some point of $\Omega$, then $S$ does not contain $A$. Since $A$ is a maximal subgroup of $G$, we have either $S A=G$ or $S \leq A$. In the former case we find that $|S|=q$, and in the latter, $|A: S|=p$, by the minimality. Since both possibilities give rise to faithful transitive permutation representations of $G$, we find that

$$
p(G)=\min \left(p^{n}, p q\right)
$$

We consider the two possibilities separately.

Case 1. $q<p^{n-1}$. Then $p(G)=p q$. This occurs for example when $q=5, p=3, n=4$.

Case 2. $q>p^{n-1}$. Then $p(G)=p^{n}$. We subdivide this into

Case $2 a . n=1$.

Case $2 b . n>1$. Then we claim that $q=\left(p^{n}-1\right) /(p-1)$. For certainly $q$ $\left(p^{n}-1\right) /(p-1)$. Suppose that $q<\left(p^{n}-1\right) /(p-1)$. Then $\left(p^{n}-1\right) /(p-1) \geq 2 q$. Hence

$$
p^{n}-1 \geq 2 q(p-1) \geq 2 p^{n-1}(p-1) \text {. }
$$

so $2 p^{n-1}-1>p^{n}$ and $p^{n}-1>p^{n}$, a contradiction. So here, $q=\left(p^{n}-1\right) /(p-1)$. An example is $p=3, n=3, q=13$.

Now we consider $q(G)$. By Lemma 3.4 and the remarks preceding it, a cyclic group $\langle c\rangle$ of order $p$ has, up to isomorphism, precisely one faithful irreducible module $M$ over 
Q. We have $\chi_{M}\left(c^{i}\right)=-1$ if $1 \leq i \leq p-1$ and $\chi_{M}(1)=p-1$. We consider the various cases above.

Suppose that $n=1$, which clearly can occur only in Case 2a. Let $V$ be a faithful quasi-permutation module over $\mathbb{Q}$ for $G$. It contains a faithful irreducible module and a trivial module, so by the above, $q(G) \geq(p-1)+1=p=p(G)$. Therefore $q(G)=p(G)$ in this case.

Now assume that $n>1$, and again let $V$ be a faithful quasi-permutation module over Q of dimension $q(G)$. We have $V=U \oplus W$ for some faithful irreducible submodule $U$ and submodule $W$. If $-k=\min _{x \in G} \chi_{U}(x)$, then $\operatorname{dim} W \geq k$. Let $U_{1}$ be an irreducible $A$-submodule of $U$, and let $K=C_{A}\left(U_{1}\right)$. Then $A / K$ is cyclic, so $K \neq 1$. Hence $N_{G}(K)=A$, and $K, K^{b}, \ldots, K^{b^{q-1}}$ are all distinct. Therefore the $A$-modules $U_{1}, U_{1} b, \ldots, U_{1} b^{q-1}$ are pairwise non-isomorphic, and we find easily that

$$
U=U_{1} \oplus \ldots \oplus U_{1} b^{q-1} .
$$

Thus $\operatorname{dim} U=(p-1) q$. Any maximal subgroup of $A$ can occur as $K$ in this situation.

The case $n>1$ subdivides into two subcases, of which the second subdivides further. Suppose first that $q=\left(p^{n}-1\right) /(p-1)$, so that we are in Case $2 \mathrm{~b}$. Here, $\operatorname{dim} U=p^{n}-1$, and as $W \neq 0$, we have $q(G)=\operatorname{dim} V \geq p^{n}=p(G)$. Thus, $q(G)=p(G)$ in this case.

Finally, suppose that $q<p^{n-1}$. This is Case 1 , and $p(G)=p q$. We have $\chi_{U}(x)=0$ if $x \notin A$, and if $x \in A$, then

$$
\chi_{U}(x)=\sum_{i=0}^{q-1} \chi_{U_{1} b^{i}}(x)
$$

Now $C_{A}\left(U_{1} b^{i}\right)=K^{b^{i}}$, and so

$$
\chi_{U_{1} b^{i}}(x)= \begin{cases}-1 & \text { if } x \notin K^{b^{i}} \\ p-1 & \text { if } x \in K^{b^{i}}\end{cases}
$$

In particular, if $x \in A$, then $\chi_{U}(x) \geq-q$.

We distinguish two possibilities. The first is that there exists $x \in A$ such that $x^{b^{i}} \notin K$ for all $0 \leq i \leq q-1$. Then $\chi_{u}(x)=-q$, and we find that $q(G)=\operatorname{dim} V \geq(p-1) q+q=$ $p q=p(G)$. Therefore $q(G)=p(G)$ in this case. If $n=2$, then $|K|=p$, and the number of conjugates of the elements of $K$ is $1+(p-1) q<p^{2}$, so this possibility arises here.

The second possibility is that, for all $x \in A$, there exists $i$ such that $x^{b^{i}} \in K$. Then for all $x \in A$, we have $\chi_{U}(x) \geq-(q-1)+(p-1)>-q$. We find that $U \oplus W$, where $W$ is a trivial module of dimension $q-1$, is a faithful quasi-permutation module of dimension less than $p q$, so $q(G)<p(G)$ in this case. We have verified using CAYLEY that this case arises for $p=7, n=3, q=19, p=13, n=3, q=61$, and a few others with $n=3$. Some others were examined with $n>3$, and for those this situation did not arise. The verification amounts to showing that there is a maximal $\mathbb{F}_{p}$-subspace $K$ of $F$ such that, with $\zeta$ acting by multiplication, every $\zeta$-orbit intersects $K$ non-trivially.

\section{REFERENCES}

1. J. J. Cannon, An introduction to the group theory language, Cayley, Computational group theory, 145-183 (Academic Press, London, 1984). 
2. L. Dornhoff, Group representation theory, Part A (Marcel Dekker, New York, 1971).

3. M. Hoffman, An invariant of finite abelian groups, Amer. Math. Monthly 94 (1987), 664-666.

4. I. M. Isaacs, Character theory of finite groups (Academic Press, New York, 1976).

5. M. A. Shahabi Shojaei, Schur indices of irreducible characters of $S L(2, q)$, Arch. Math. 40 (1983), 221-231.

6. W. J. Wong, Linear groups analogous to permutation groups. J. Austral. Math. Soc. (Sec. A) 3 (1963), 180-184.

7. W. J. Wong, On linear p-groups, J. Austral. Math. Soc. (Ser. A) 4 (1964), 174-178.

\author{
J. M. BURNS: \\ Department of Mathematics \\ UNIVERSITY COLLEgE \\ GALWAY \\ IRELAND \\ B. Hartley and R. SANDLING: \\ Department of MATHEMatics \\ UNIVERSITY OF MANCHESTER \\ MANCHESTER M13 9PL \\ ENGLAND
}

\author{
B. GoldsMITH: \\ Department of Mathematics, Statistics and \\ COMPUTER SCIENCE \\ Dublin InSTITUTE OF TECHNOLOGY \\ Kevin STREeT \\ DUBlin 8 \\ IRELAND
}

